\title{
Capsule Commentary on Genoff et al., Navigating Language Barriers: A Systematic Review of Patient Navigators' Impact on Cancer Screening for Limited English Proficient Patients
}

\author{
Amy Linsky, MD, MSc ${ }^{1,2}$ \\ 'Section of General Internal Medicine, VA Boston Healthcare System, Boston, MA, USA; ${ }^{2}$ General Internal Medicine, Boston University School of \\ Medicine, Boston, MA, USA.
}

$J$ Gen Intern Med 31(4):415

DOI: $10.1007 / \mathrm{s} 11606-016-3602-9$

(๑) Society of General Internal Medicine 2016

$\mathrm{T}$ he study by Genoff et al. reviews the current literature to summarize what is known - and importantly, what is unknown - about the use of patient navigators to improve cancer screening rates for patients with limited English proficiency (LEP). ${ }^{1}$ They identified 15 articles meeting their inclusion criteria, 14 of which found that use of navigators increased screening for breast, cervical, or colorectal cancer. This adds to the literature supporting the use of navigators for lower income patients and to the importance of culturally sensitive care. ${ }^{2,3}$

However, this is also where multiple factors seem to get tangled. As highlighted by the authors of this review, the patient navigator interventions were very diverse, making it difficult to determine the most effective components. While focusing primarily on language barriers, there is no acknowledgment of the language spoken by the clinical provider. Since language concordance between patients and providers does not reduce disparities, as seen previously, this would suggest that the language itself is not the issue. ${ }^{4}$ Second, some interventions included an educational component while others did not. The relative importance of each of these is unclear. Another confounder is the role of socioeconomic status and the barriers to access often seen in those with lower income. Finally, it may be that the improvement in screening is related to culturally tailored care, as several of the studies used navigators from the same community from which patients were drawn. This is similar to improved relationships between

This comment refers to the article available at: $h t t p: / / d x . d o i . o r g / 10.1007 /$ s11606-015-3572-3.

Published online February 8, 2016 patients and providers who have concordant backgrounds. ${ }^{5}$ Taken together, the interaction between language, education, socioeconomic status, and culture on screening rates is complicated and difficult to separate.

That said, in the end, the use of navigators does improve rates of preventive cancer screenings. The essential next step is to identify the key components for success so they can be replicated and integrated in a more systematic method. This way, limited financial resources can be directed to those aspects of navigation that yield the highest value in terms of health outcomes and patient satisfaction.

Corresponding Author: Amy Linsky, MD, MSc; Section of General Internal MedicineVA Boston Healthcare System, Boston, MA 02130, USA (e-mail: amy.linsky@va.gov).

\section{Compliance with ethical standards:}

Conflict of interest: The author has no conflicts of interest with the material in this article.

\section{REFERENCES}

1. Genoff MC, Zaballa A, Gany F, Gonzalez J, Ramirez J, Jewell ST, Diamond L. Navigating language barriers: a systematic review of patient navigators' impact on cancer screening for limited English proficient patients. JGIM. 2016. doi:10.1007/s11606-015-3572-3.

2. Paskett ED, Harrop JP, Wells KJ. Patient navigation: an update on the state of the science. CA: Cancer J Clin. 2011;61(4):237-49.

3. Tucker CM, Arthur TM, Roncoroni J, Wall w, Sanchez J. Patientcentered, culturally sensitive health care. Am J Lifestyle Med. 2015;9(1):63-77.

4. Eamranond PP, Davis RB, Phillips RS, Wee CC. Patient-physician language concordance and primary care screening among Spanishspeaking patients. Med Care. 2011;49(7):668-72.

5. Street RL Jr, O'Malley KJ, Cooper LA, Haidet P. Understanding concordance in patient-physician relationships: personal and ethnic dimensions of shared identity. Ann Fam Med. 2008;6(3):198-205. 